\title{
The Importance of Weight Change Experiences for Performance of Diabetes Self-Care: A Patient- Centered Approach to Evaluating Clinical Outcomes in Type 2 Diabetes
}

Shana B. Traina $\cdot$ April Slee $\cdot$ Sangsoon Woo $\cdot$ William Canovatchel

To view enhanced content go to www.diabetestherapy-open.com

Received: September 10, 2015 / Published online: November 25, 2015

(C) The Author(s) 2015. This article is published with open access at Springerlink.com

\section{ABSTRACT}

Introduction: The aim of this study was to examine the influence of weight change experiences over time on motivation to perform diabetes self-care behaviors using data from a study of canagliflozin (an agent that inhibits sodium glucose co-transporter 2) versus glimepiride in dual therapy with metformin and background diet/exercise.

Methods: Weight and motivation for performing healthy behaviors were collected at baseline and over time. The motivation questionnaire enabled categorization into two groups: those performing or not performing health behaviors. Four distinct patterns of weight change were determined: losing weight, gaining weight, and two patterns for

Electronic supplementary material The online version of this article (doi:10.1007/s13300-015-0145-8) contains supplementary material, which is available to authorized users.

S. B. Traina $(\bowtie) \cdot$ W. Canovatchel Janssen Global Services, LLC, Raritan, NJ, USA e-mail: straina@its.jnj.com

A. Slee $\cdot$ S. Woo

Axio Research, Seattle, WA, USA fluctuating weight. The relationships between these patterns and motivation for weight loss, following a diet, and exercise were examined using logistic regression models.

Results: Of 1182 subjects, more than half were already performing behaviors to lose weight, diet, and exercise at baseline. Among those who were not, 52\% (246/474) started taking action to lose weight after baseline, 54\% (241/448) started following a diet, and 42\% (232/556) started exercising. Weight change patterns were significantly related to performance of healthy behaviors at follow-up (week 36). Compared to the weight gain pattern, those who experienced a continuous weight loss pattern from baseline to week 36 were 2.2 (95\% confidence interval $1.49,3.37)$ times more likely to perform the healthy behaviors. Baseline behavior and confidence were also predictive of performing healthy behaviors.

Conclusion: The current work highlights the importance of weight change patterns for performance of diabetes self-care. Tracking weight patterns over time, assessing confidence for performance of healthy behaviors, and being aware of the relationship between weight changes and diabetes self-care 
behaviors are viable, concrete ways to practice patient-centered care.

Funding: Janssen Global Services, LLC.

Keywords: Behavior; Patient-reported outcomes; Self-care; Type 2 diabetes; Weight

\section{INTRODUCTION}

Type 2 diabetes is primarily a self-managed disease. Self-care behaviors, such as following a healthy diet, being physically active, taking medications as prescribed, and monitoring health (e.g., glucose levels, body weight, blood pressure) [1], are an important part of achieving optimal outcomes and can have a major impact on the lives of patients, their families, and society [2]. For a person to adhere to self-care activities, he or she must have the knowledge and skill to perform these activities, the confidence (i.e., self-efficacy [3]) and motivation to do so, and the belief that performance will be beneficial for managing the disease. Determinants of self-care include cultural [4], psychosocial [5], physical [6], and economic [7] aspects. Treatments can also influence behavior through their impact on factors such as glucose control, weight, blood pressure, and hypoglycemia. Experiencing benefits can be encouraging and may lead to increased performance of healthy behaviors, thus continuing the cycle of realized benefits and reinforcing the behaviors. Conversely, drawbacks associated with treatments may deter performance of healthy behaviors.

Continuous reinforcement of healthy behaviors, as well as continued or maintained weight loss, has been shown to increase motivation [8]. Motivational Interviewing (MI) is a directive, person-centered intervention designed to explore ambivalence/resistance to behavior change, support self-efficacy, and activate motivation to perform target behaviors in a nonjudgemental way $[9,10]$. Studies have shown that MI can contribute to improvements in self-management abilities and outcomes among people with type 2 diabetes [3, 11].

MI has been employed by health professionals to assess a person's readiness to make a behavioral change (RTC) and to help people maintain performance of diabetes self-care behaviors. Five stages of RTC are described by the transtheoretical model (TTM) [12]. The TTM focuses on the decision-making of the individual and is a model of intentional change. The TTM posits that individuals move through five stages of change: precontemplation (e.g., a healthier lifestyle is not yet considered), contemplation (e.g., thinking about behavior change), preparation (e.g., intending to but not yet taking action), action (e.g., starting to modify behaviors in the last 6 months), and maintenance (e.g., stabilizing behavior change and avoiding relapse over $>6$ months). For each stage of change, different intervention strategies are likely more effective in moving the person to the next stage of change and ultimately to maintenance [13]. The TTM dichotomizes the five stages of RTC into pre-Action (i.e., precontemplation, contemplation, and preparation) and Action (i.e., action and maintenance) stages [14].

The TTM assumes that behavior changes are cyclical. This means that people living with type 2 diabetes can move forward and backward through the stages of RTC. This is often referred to as recycling, which can occur multiple times before changing for good. Recycling is not failing, but a natural occurrence that may be helpful in inducing lifelong behavioral changes, since individuals tend to have a heightened focus on barriers or challenges during these 
times. People can simultaneously be at different RTC stages for individual behaviors, such as engaging in weight loss/maintenance, healthy eating, and exercise. Assessing the stage of change for each target behavior is particularly important for guiding and evaluating interventions [15]. Research has indicated that interventions tailored to the individual's stage of change work better than generic approaches [16]. Moving to the Action stages of RTC for diabetes-specific behaviors has been related to reductions in HbA1c [17].

Findings from qualitative interviews among people with type 2 diabetes suggest that individual experiences are complex and influenced by cognitive, behavioral, and emotional dimensions that are linked with all aspects of peoples' daily lives [18]. How people feel about their weight has been posited to be an important driver of behavior. A recent empirical study showed that satisfaction with weight was strongly associated with positive behaviors and outcomes [19]. In our previous work, we found that weight loss led to improvements in self-reported weight-related quality of life and satisfaction with health, which are associated with consistent and persistent performance of healthy behaviors [20]. In that study, for simplicity, participants were classified into two groups: $<5$ or $\geq 5$ pounds of weight loss [20]. Note, any threshold chosen is not meaningful for all people; what one person perceives as a meaningful change may not be meaningful to another. In addition, different patterns of response may produce the same change over time, but very different experiences. For example, losing 5 pounds and then gaining it back for a net change of zero is a different experience than never having changed weight. The experience of regaining weight may be more discouraging than maintaining the same weight. The insights gained from our previous study [20] highlight the need to shift focus from "clinically meaningful" weight loss (i.e., sufficient weight loss to impact a clinical outcome) to the patient experience of weight loss, which is more relevant for patient-reported outcomes. Defining arbitrary thresholds for weight loss is not useful when the goal is to describe the amount of weight loss that will motivate an individual to adopt and maintain healthy behaviors. Furthermore, understanding which weight change pattern a person has experienced is important for encouraging target behaviors.

However, it is challenging to get a clear picture of a person's experience with weight change because the methods used in current practice typically rely on weight measurements at clinic visits that are several months apart. Measurement of net weight change does not take into account trends and experiences over time; between visits, a person may have experienced noticeable weight gains and/or losses. When considering randomized controlled trial results to assist in treatment selection, it is important to recognize that reported mean changes do not address the heterogeneity of treatment effects, or trends over time [21].

The current study explores the empirical relationship between weight change experiences and RTC behavior, using an approach designed to better describe individual weight experiences.

\section{METHODS}

\section{Data Set}

This analysis is based on results from a 104-week, randomized, double-blind, Phase 3 study that assessed the efficacy and safety of canagliflozin 100 and $300 \mathrm{mg}$ versus maximally 
tolerated doses of glimepiride in subjects with type 2 diabetes inadequately controlled with metformin and background diet and exercise $[22,23]$. Canagliflozin (an agent that inhibits sodium glucose co-transporter 2 [SGLT2]) has been shown to significantly reduce $\mathrm{HbA1c}$, body weight, and blood pressure. Canagliflozin has been associated with adverse events (AEs) related to the mechanism of action (e.g., genital mycotic infections, urinary tract infections, and AEs related to volume depletion and osmotic diuresis); however, canagliflozin has been shown to be well tolerated, as these events led to few discontinuations in the clinical trial program [22, 23]. Glimepiride has also been shown to significantly reduce HbA1c; however, it has been associated with weight gain and an increase in hypoglycemia [2]. In this trial, canagliflozin $300 \mathrm{mg}$ demonstrated superiority, and canagliflozin $100 \mathrm{mg}$ demonstrated noninferiority, to glimepiride in HbA1c lowering at 52 weeks [22]; these effects were maintained at 104 weeks [23]. Both canagliflozin doses were associated with a mean weight loss of $3.6 \mathrm{~kg}$ and glimepiride was associated with a mean weight gain of $0.8 \mathrm{~kg}$ at week 104 [23].

Weight, RTC behavior, the effect of weight on quality of life, and self-efficacy for healthy behaviors were measured at baseline and over time. Specifically, body weight was measured at baseline and during clinic visits at weeks 4,8 , $12,18,26,36,44$, and 52. RTC behavior was measured at baseline and weeks 4,8 , and 36 using individual items that were developed to identify a respondent's TTM stage of change for weight loss, exercise, and following a diet. For each self-care behavior, five response options were provided to map the five stages of RTC (see Fig. S1 in the supplementary material). The effect of weight on quality of life was measured using the Impact of Weight on Quality of
Life-Lite (IWQoL-Lite) questionnaire [24] at baseline and week 52 . This instrument assesses the impact of weight on several domains of quality of life, including physical function, self-esteem, sexual life, public distress, and work, which are measured on a scale from 0 (highest burden) to 100 (lowest burden). Self-efficacy for healthy behaviors was measured using the Multidimensional Diabetes Questionnaire (MDQ) at baseline and weeks 4, 8 , and 36. This instrument captures self-efficacy for the ability to maintain a healthy weight, follow a diet, and resist food temptations [25]. For simplicity, scales were dichotomized above and below the midpoint, where scores above the midpoint indicate a greater degree of self-efficacy. For weight loss and exercise, self-efficacy was measured using only one item each. For diet, two items that related to following a diet and resisting food temptations were combined (>midpoint on both items versus >midpoint on zero or one item). A check box was provided for respondents to indicate whether they were advised not to exercise; we used this to exclude subjects from the exercise behavior outcome analyses.

\section{Weight Change Patterns}

Randomized clinical trials often summarize the effect of an intervention on weight using mean within-patient change values to describe the change from baseline to subsequent time points. However, this calculation does not reflect individual experiences of weight change over time, making it difficult for health care professionals to apply this to individuals. Also, perceptions of weight change are not necessarily linear. Lane et al. [26] showed that weight gain had a larger negative impact on people with type 2 diabetes than the positive impact of the same amount of weight loss. 
To determine patterns of weight change in individuals, a clustering algorithm [27] was applied to pooled, individual weight change data over time from three trials (not including the trial that was analyzed in this report) [28-30]. Additional details are provided in Appendix 1 in the supplementary material. This analysis suggested that subjects experienced steady weight gain, steady weight loss, initial weight loss followed by weight gain, or initial weight gain followed by weight loss. As a result of these findings, we defined the experience of weight change over time using four patterns, as follows:

- Pattern 1: lost any weight from baseline to week 18, and lost any weight from week 18 to 36 ;

- Pattern 2: lost any weight from baseline to week 18, and gained any weight or did not change weight from week 18 to 36;

- Pattern 3: gained any weight or did not change weight from baseline to week 18 , and lost any weight from week 18 to 36;

- Pattern 4: gained any weight or did not change weight from baseline to week 18 , and gained any weight or did not change weight from week 18 to 36 .

Additional details on the derivation of these patterns are provided in Appendix 1 in the supplementary material.

\section{Statistical Methods}

The primary outcome was 'Action' stage versus 'pre-Action' stage for each self-care behavior at week 36. Univariate associations between baseline sociodemographic, clinical, and patient-reported characteristics and RTC stage were compared using the $t$ test or chi-square test, as appropriate. The relationships between weight change patterns and maintenance of or movement into the Action stage of RTC at week
36 were examined for three health behaviors (engaging in weight loss, following a diabetes diet, and exercise) using logistic regression models. Baseline RTC and self-efficacy were included because previous work has shown that past behavior predicts future behavior [31], and self-efficacy has been linked to performance of behaviors [3]. Other covariate adjustments included age, gender, race, baseline body mass index (BMI), and baseline HbA1c (\%). Smoking status and history of depression were included, as these factors may impact the performance of the health behaviors of interest. Overall functional status was accounted for with the Short-Form 36 (SF-36) physical and mental component summary scores [32]; weight-related self-esteem was included as a continuous covariate and weight-related physical function was dichotomized into those who were in the lowest quartile (most impaired) versus the top three quartiles.

\section{RESULTS}

Of 1182 subjects with RTC data at baseline and week 36, more than half were taking Action for weight loss, diet, and exercise at baseline, respectively ( $n=1174$ for exercise; excludes eight who were advised not to exercise). At baseline, $32 \%$ of subjects were taking Action for all three behaviors and $16 \%$ were not taking Action for any of the three behaviors. Those taking Action for weight loss at baseline were more likely to be male, less likely to be Caucasian, and had higher self-efficacy for all three self-care behaviors. Among those already taking Action for weight loss, $71 \%$ were also taking Action for following a diet and $66 \%$ were also taking Action for exercise. Those taking Action for diet at baseline were similar, but additionally had a lower average BMI and better 
health-related quality of life. The greatest difference in BMI was observed between baseline Action and pre-Action for exercise. HbA1c only differed by baseline RTC for diet, but not for weight loss or exercise (Table 1).

For each behavior, the net proportion of subjects in Action increased between baseline and week 36 by about $10 \%$ (Table 2). Relationships between Action and pre-Action at week 36 and demographic, clinical, and patient-reported variables were generally similar to associations observed at baseline. Among subjects randomized to canagliflozin, $72 \%$ were taking Action for weight loss at week 36 versus $65 \%$ for glimepiride $(P=0.015)$. Among subjects randomized to canagliflozin, $78 \%$ were taking Action for diet at week 36 versus $72 \%$ for glimepiride $(P=0.051)$. For exercise, the proportion taking Action at week 36 was not statistically different by treatment assignment (67\% and 64\%, respectively).

In examining body weight through week 36 , it was observed that $40 \%$ of subjects experienced weight change Pattern 1, 32\% experienced Pattern 2, 13\% experienced Pattern 3, and 16\% experienced Pattern 4 (Fig. 1). On average, those in weight change Pattern 1 initially lost about $3.4 \mathrm{~kg}$ and then lost another $2.0 \mathrm{~kg}$; the average net loss was $5.3 \mathrm{~kg}$. The Pattern 2 group initially lost about $3.5 \mathrm{~kg}$ and then regained about half, resulting in a net loss of $2.1 \mathrm{~kg}$. The Pattern 3 group initially gained about $1.8 \mathrm{~kg}$ and then lost $1.6 \mathrm{~kg}$, resulting in a net gain of $0.2 \mathrm{~kg}$. The Pattern 4 group gained $3.3 \mathrm{~kg}$ on average, about half during the first 18 weeks and half during the second 18 weeks. Among those randomized to canagliflozin, $52 \%$ and $4 \%$ experienced weight change Patterns 1 and 4, compared to $13 \%$ and $40 \%$ for glimepiride, respectively ( $P$ value comparing distributions $<0.001$ ).

\section{Regressions}

\section{Weight Loss}

Weight change pattern was significantly related to taking Action for weight loss at week 36 (Table 3). Subjects who experienced Patterns 1 and 2 were 2.2 and 1.7 times more likely to take Action for weight loss at week 36, respectively, versus those in the Pattern 4 group. Consistent with previous research [31], baseline RTC was an important predictor of future behavior (odds ratio [OR] 4.4). Interestingly, self-efficacy was statistically significant even after controlling for baseline RTC. Baseline BMI, race, and weight-related self-esteem were also significantly related to taking Action for weight loss as week 36. The OR for baseline HbA1c was numerically less than $1(P=0.052)$, suggesting lower HbA1c values may be related to taking Action. Lower weight-related self-esteem was related to taking Action, which may reflect the need for some level of negative impact of weight on self-esteem to necessitate Action.

\section{Diet}

Weight change pattern was significantly related to taking Action for diet at week 36 (Table 3). Compared to the Pattern 4 group, those who experienced Pattern 1 were 1.9 times more likely to follow a diet. Baseline RTC was an important predictor of taking Action for diet at week 36 (OR 6.66). Women were 1.4 times more likely to take Action for diet versus men, and smokers were significantly less likely to take Action for diet. Self-efficacy for following a diet and resisting food temptations was related to being in Action for diet at week 36.

\section{Exercise}

Subjects who experienced Pattern 1 were 2 times more likely to be taking Action for exercise compared to those who experienced 


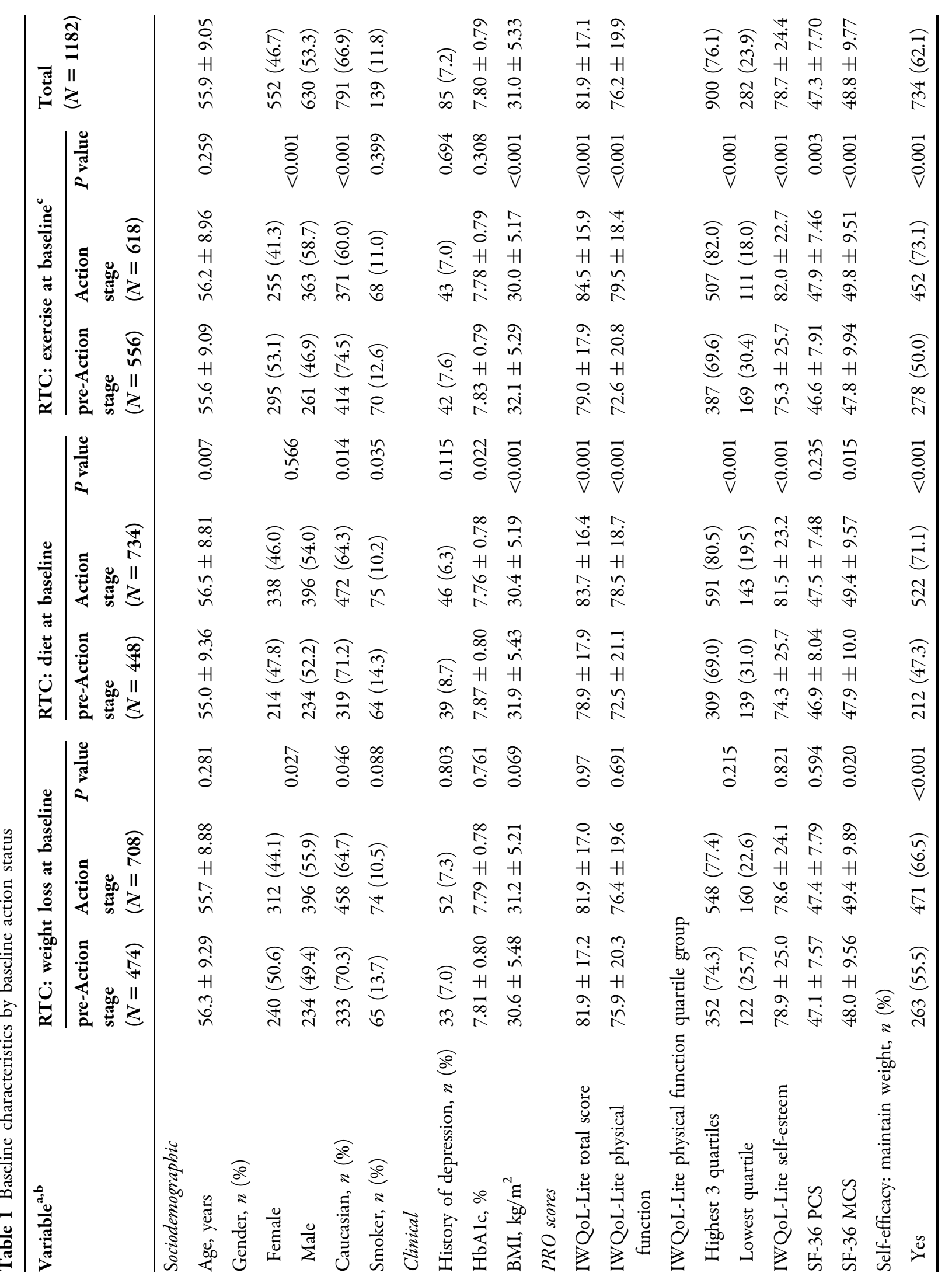




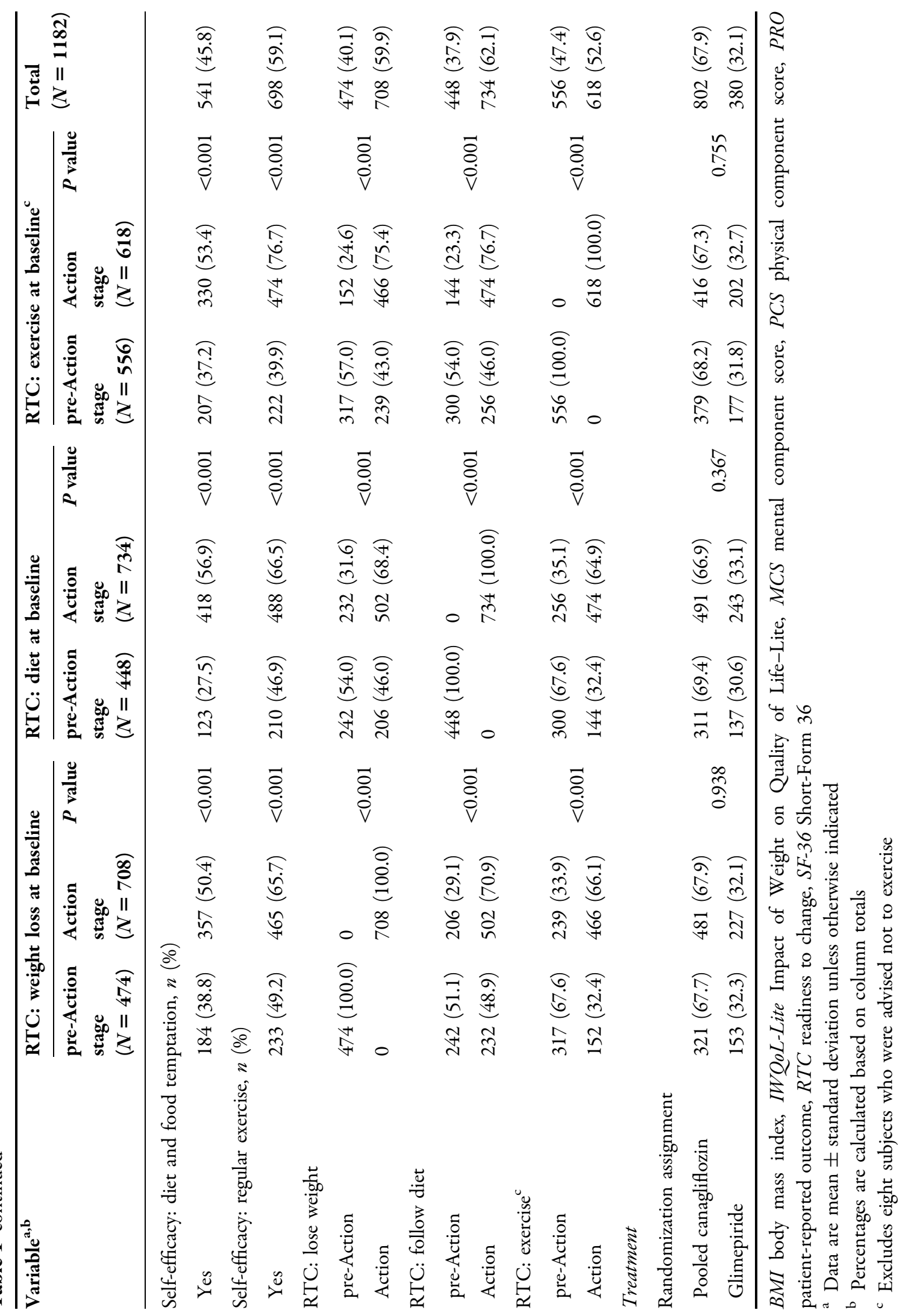


Table 2 Number (\%) of subjects who were taking action for weight loss, diet, and exercise at baseline and week 36

\begin{tabular}{lcccccc}
\hline Baseline & \multicolumn{1}{c}{ Week 36 } & & \\
\cline { 2 - 6 } & pre-Action & Action & Total \\
\hline Taking Action to lose weight $(N=1182)$ \\
pre-Action & 228 & $19 \%$ & 246 & $21 \%$ & 474 & $40 \%$ \\
Action & 127 & $11 \%$ & 581 & $49 \%$ & 708 & $60 \%$ \\
Total & 355 & $30 \%$ & 827 & $70 \%$ & 1182 & $100 \%$ \\
Taking Action to follow diet $(N=1182)$ & & \\
pre-Action & 207 & $18 \%$ & 241 & $20 \%$ & 448 & $38 \%$ \\
Action & 78 & $7 \%$ & 656 & $55 \%$ & 734 & $62 \%$ \\
Total & 285 & $24 \%$ & 897 & $76 \%$ & 1182 & $100 \%$ \\
Taking Action to exercise $(N=1174)$ & & \\
pre-Action & 324 & $28 \%$ & 232 & $20 \%$ & 556 & $47 \%$ \\
Action & 79 & $7 \%$ & 539 & $46 \%$ & 618 & $53 \%$ \\
Total & 403 & $34 \%$ & 771 & $66 \%$ & 1174 & $100 \%$ \\
\hline
\end{tabular}

Pattern 4 (Table 3). As for the other self-care behaviors, baseline RTC was an important predictor of taking Action; taking Action for exercise at baseline was related to an eight-fold increase for taking Action at week 36. Despite controlling for this relationship, self-efficacy for exercise was a significant predictor of taking Action.

\section{Compliance with Ethics Guidelines}

The analysis in this article is based on previously conducted studies and does not involve any new studies of human or animal subjects performed by any of the authors.

\section{DISCUSSION}

Weight loss is one of the healthy behaviors recommended by the American Diabetes Association for successful type 2 diabetes management [1], but motivating patients to lose weight and perform other healthy behaviors is challenging. Part of the problem in motivating people to perform healthy behaviors is the lack of a perceivable relationship between these day-to-day activities and events that may occur many years in the future [33].

In spite of these challenges, some patients do lose weight. While weight loss and weight maintenance among people with type 2 diabetes is infrequent in everyday clinical practice, new classes of antihyperglycemic medications, such as SGLT2 inhibitors, have been shown to cause weight loss in randomized controlled trials through their mechanism of action (i.e., patients experience a net caloric loss of approximately $400 \mathrm{kcal} /$ day as a result of increased urinary glucose excretion) [28-30]. The majority of weight loss with SGLT2 inhibitors is experienced in the first 12 weeks after initiation of treatment [23]. However, some people continue to lose weight over time, and others are able to maintain a healthy weight long after 12 weeks [23]. For these people, the psychological effect of the initial weight loss may be different than for those who do not continue to lose or maintain weight. In this study, for about $90 \%$ of subjects, the first report of action was prior to or concurrent with the first recorded weight loss for all actions explored. Thus, we do not believe that the action typically precedes weight loss, but more frequent measurement would be required to clarify the temporal relationship.

The current work highlights the importance of weight change patterns for performance of diabetes self-care. Because some therapies have been associated with weight gain while others are considered weight neutral, and more recently approved options are associated with weight loss [34], treatment choice can influence weight change patterns. When a therapy 

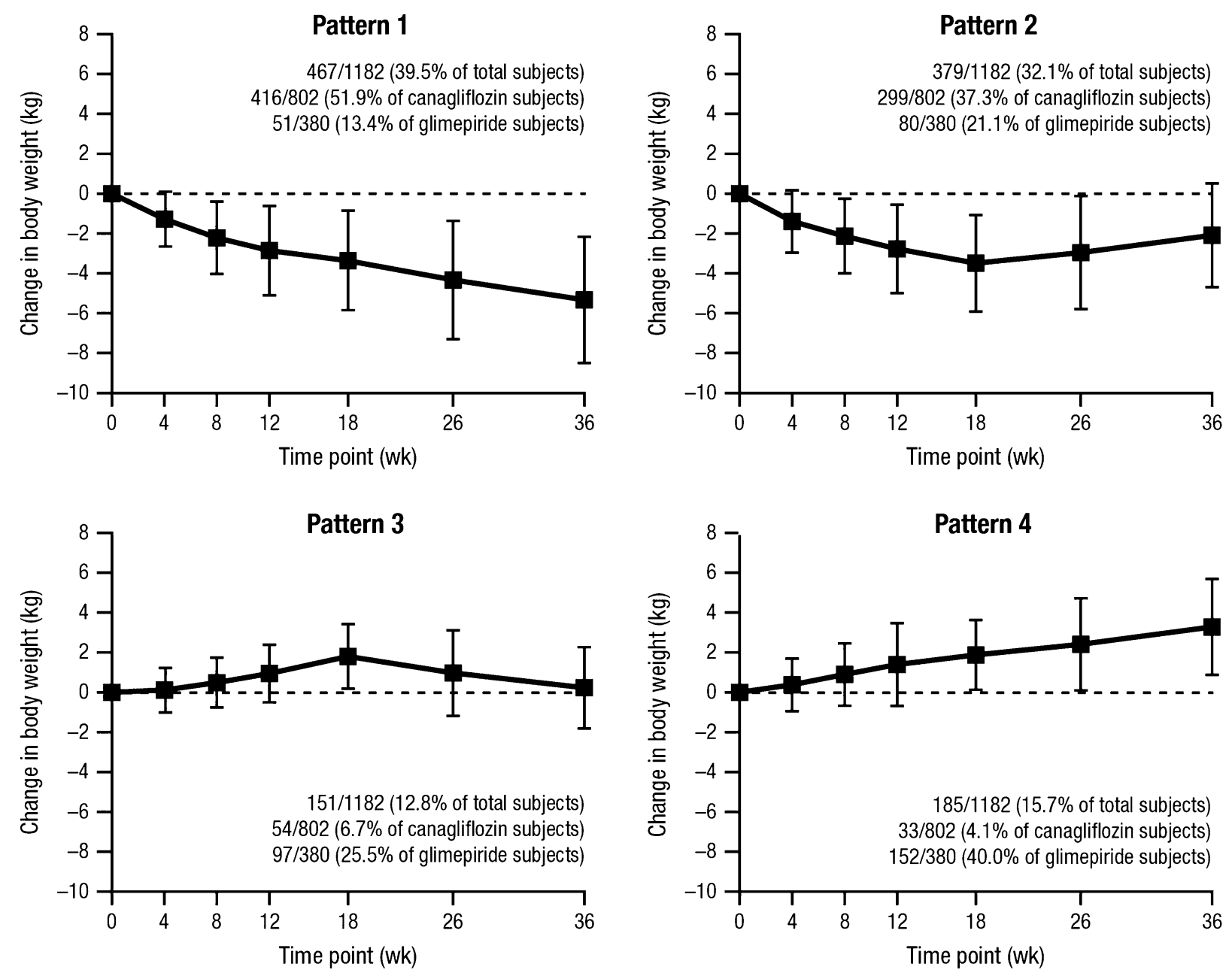

Fig. 1 Change in weight from baseline by weight pattern. Data are mean \pm standard deviation from baseline

associated with weight gain is prescribed, additional support that encourages self-care behaviors may be required to mitigate this effect.

Clinical measures that are important for the management of type 2 diabetes are increasingly being evaluated based on trajectories or patterns [35-37]. For example, studies by Feldstein et al. have shown that weight loss trajectories after diagnosis of type 2 diabetes are associated with improvements in glycemic and blood pressure control, while higher stable weight and weight gain patterns are associated with poorer glycemic and blood pressure outcomes [35,
36]. Walraven et al. [37] tracked HbA1c trajectories and observed that unfavorable glycemic outcomes were more common among younger patients and among those with higher baseline HbA1c levels and longer duration of diabetes.

Although patterns of change in clinical variables have been examined, our motivation for defining weight change patterns was to more appropriately reflect the experience of people living with type 2 diabetes. In addition to considering weight as a risk factor for future micro- and macrovascular complications, we hope that health care professionals will also 
Table 3 Relationship between weight, baseline covariates, and readiness to change at week 36

\begin{tabular}{|c|c|c|c|c|c|c|c|c|c|}
\hline & \multicolumn{3}{|c|}{ Lose weight } & \multicolumn{3}{|c|}{ Follow diet } & \multicolumn{3}{|c|}{ Exercise } \\
\hline & $\overline{\text { OR }}$ & $95 \% \mathrm{CI}$ & $P$ value & $\overline{\mathrm{OR}}$ & $95 \% \mathrm{CI}$ & $P$ value & $\overline{\mathrm{OR}}$ & 95\% CI & $P$ value \\
\hline \multicolumn{10}{|l|}{ Weight trajectories } \\
\hline Pattern 1 (vs. Pattern 4) & 2.24 & $1.49,3.37$ & & 1.92 & $1.23,3.02$ & & 2.01 & $1.31,3.09$ & \\
\hline Pattern 2 (vs. Pattern 4) & 1.73 & $1.14,2.61$ & $<0.001$ & 1.30 & $0.83,2.04$ & 0.003 & 1.34 & $0.86,2.07$ & 0.005 \\
\hline Pattern 3 (vs. Pattern 4) & 1.16 & $0.71,1.89$ & & 0.88 & $0.51,1.51$ & & 1.17 & $0.69,1.99$ & \\
\hline Self-efficacy (vs. no self-efficacy) ${ }^{a}$ & 1.64 & $1.21,2.23$ & 0.001 & 1.93 & $1.38,2.69$ & $<0.001$ & 2.11 & $1.56,2.85$ & $<0.001$ \\
\hline Action (vs. pre-Action) ${ }^{a}$ & 4.40 & $3.32,5.84$ & $<0.001$ & 6.66 & $4.83,9.19$ & $<0.001$ & 7.96 & $5.81,10.90$ & $<0.001$ \\
\hline Age ( 1 year) & 1.00 & $0.99,1.02$ & 0.738 & 1.00 & $0.98,1.02$ & 0.864 & 0.99 & $0.98,1.01$ & 0.379 \\
\hline Female (vs. male) & 1.22 & $0.90,1.63$ & 0.197 & 1.40 & $1.01,1.94$ & 0.046 & 0.97 & $0.71,1.31$ & 0.840 \\
\hline Caucasian race (vs. non-Caucasian) & 0.58 & $0.41,0.83$ & 0.003 & 1.08 & $0.74,1.57$ & 0.694 & 1.02 & $0.71,1.47$ & 0.897 \\
\hline Smoker (vs. non-smoker) & 1.09 & $0.71,1.67$ & 0.680 & 0.62 & $0.40,0.96$ & 0.031 & 0.74 & $0.48,1.15$ & 0.184 \\
\hline BMI (1 unit $)^{a}$ & 1.09 & $1.05,1.13$ & $<0.001$ & 1.02 & $0.98,1.05$ & 0.385 & 1.00 & $0.96,1.03$ & 0.785 \\
\hline HbAlc $(\text { per } 1 \%)^{a}$ & 0.84 & $0.71,1.00$ & 0.052 & 0.95 & $0.79,1.16$ & 0.632 & 1.07 & $0.89,1.29$ & 0.450 \\
\hline $\begin{array}{l}\text { History of depression (vs. no } \\
\text { history) }\end{array}$ & 0.83 & $0.48,1.43$ & 0.500 & 0.63 & $0.36,1.11$ & 0.109 & 0.83 & $0.47,1.47$ & 0.527 \\
\hline $\begin{array}{l}\text { Weight-related self-esteem } \\
(1 \text { point })^{\mathrm{b}}\end{array}$ & 0.82 & $0.66,1.03$ & 0.087 & 1.06 & $0.85,1.33$ & 0.604 & 0.97 & $0.78,1.20$ & 0.788 \\
\hline $\begin{array}{l}\text { Weight-related physical function } \\
\text { lowest quartile (vs. higher) }{ }^{\mathrm{b}}\end{array}$ & 0.82 & $0.55,1.23$ & 0.347 & 1.07 & $0.71,1.64$ & 0.739 & 0.80 & $0.54,1.20$ & 0.283 \\
\hline General mental functional status $^{c}$ & 0.99 & $0.97,1.00$ & 0.103 & 1.00 & $0.98,1.02$ & 0.891 & 1.00 & $0.99,1.02$ & 0.885 \\
\hline General physical functional status ${ }^{c}$ & 0.99 & $0.97,1.01$ & 0.371 & 1.00 & $0.97,1.02$ & 0.781 & 0.99 & $0.97,1.01$ & 0.540 \\
\hline
\end{tabular}

$B M I$ body mass index, $C I$ confidence interval, $O R$ odds ratio

${ }^{a}$ Measured at baseline

b Based on Impact of Weight on Quality of Life-Lite self-esteem and physical function subscales

${ }^{c}$ Based on the Short-Form 36 physical component score and mental component score

understand weight as a process that people with type 2 diabetes are experiencing and using to make decisions about whether they will perform diabetes self-care behaviors.

Because longitudinal weight data are collected in clinical practice, health care professionals can look back further than the last visit to get an overall view of weight patterns (as in pediatrics). Accurate determination of weight patterns could be supplemented by the addition of self-reported weight information in between visits. The increasing popularity of health-tracking applications could be leveraged in this capacity. Speaking with people about personal data that they may be tracking may improve responsiveness to suboptimal results and expedite adjustments to treatments and/or the 
need to provide additional diabetes management support and education. In addition, exploring how people feel about their weight pattern may help to operationalize the central role of the patient in collaborative disease management efforts.

An important result of this work is the identification of a relationship between self-efficacy and behaviors, despite adjustment for prior behaviors. The obvious risk for those already engaging in healthy behaviors is that they might stop. Therefore, it is important to commend and encourage persistent and consistent performance of self-care so that people can appreciate the link between their behaviors and outcomes [8]. MI techniques can help facilitate the identification of specific behaviors where self-efficacy may be higher to guide collaboration and planning for performance of these target behaviors $[3,11]$.

We focused on weight loss and not treatment effect, since our goal was to describe how weight loss might impact future behaviors. Because the primary mechanism of action of SGLT2 inhibitors is to cause excretion of excess glucose in urine, the glycemic efficacy of canagliflozin is linked to the causal pathway for weight loss. While the results of Phase 3 trials showed overwhelmingly greater weight loss with canagliflozin compared with placebo and active comparators, many individuals in the comparator arms did lose weight during the studies [22, 23, 28-30]. We acknowledge that one limitation of this study is that the amount of weight change experienced by the people in this sample is not typical in real-world settings; therefore, the patterns of weight over time in a group of people without an intervention could be different.

'Patient-centered care' has become part of the lexicon for the management of diabetes [2].
The American Diabetes Association/European Association for the Study of Diabetes guidelines note, "Ultimately, it is patients who make the final decisions regarding their lifestyle choices and, to some degree, pharmaceutical interventions they use; their implementation occurs in the context of the patients' real lives and relies on the consumption of resources (both public and private) [2]." This work provides empirical support for some small, but meaningful, patient-centered actions that practitioners can take today. Specifically, these include tracking weight change patterns over time, assessing self-efficacy for healthy behaviors, and being aware of the relationship between weight changes and diabetes self-care behaviors.

\section{ACKNOWLEDGMENTS}

This analysis was funded by Janssen Global Services, LLC, and was based on data from a study funded by Janssen Research and Development, LLC. The article processing charges for this publication were funded by Janssen Global Services, LLC. Editorial support was provided by Cherie Koch, PhD, of MedErgy, and was funded by Janssen Global Services, LLC. Canagliflozin has been developed by Janssen Research \& Development, LLC, in collaboration with Mitsubishi Tanabe Pharma Corporation. S.B.T. is the guarantor of this work, had full access to the data, and takes responsibility for the integrity of the data and the accuracy of the analyses. The authors acknowledge Cheryl Neslusan, PhD, of Janssen Global Services, LLC, for her contributions to data interpretation and critical revision of the manuscript. All named authors meet the International Committee of Medical Journal 
Editors (ICMJE) criteria for authorship for this manuscript, take responsibility for the integrity of the work as a whole, and have given final approval for the version to be published.

Author contribution. S.B.T. contributed to the conception and design of the analysis; the acquisition, analysis, and interpretation of the data; and the development of the manuscript. A.S. contributed to the conception and design of the analysis; the analysis and interpretation of the data; and the development of the manuscript. S.W. contributed to the analysis and interpretation of the data and the development of the manuscript. W.C. contributed to the acquisition and interpretation of the data and the development of the manuscript. All authors approved the final version of the submitted manuscript.

Disclosures. S.B.T. and W.C. are full-time employees of Janssen Global Services, LLC. A.S. and S.W. are full-time employees of Axio Research, and they received payment from Janssen for statistical support of the analyses reported in this manuscript.

Compliance with ethics guidelines. The analysis in this article is based on previously conducted studies and does not involve any new studies of human or animal subjects performed by any of the authors.

Open Access. This article is distributed under the terms of the Creative Commons Attribution-NonCommercial 4.0 International License (http://creativecommons.org/licenses/ by-nc/4.0/), which permits any noncommercial use, distribution, and reproduction in any medium, provided you give appropriate credit to the original author(s) and the source, provide a link to the Creative Commons license, and indicate if changes were made.

\section{REFERENCES}

1. American Association of Diabetes Educators Position Statement. AADE7 self-care behaviors. Diabetes Educ. 2008;34:445-9.

2. Inzucchi SE, Bergenstal RM, Buse JB, Diamant $M$, Ferrannini E, Nauck $M$, et al. Management of hyperglycemia in type 2 diabetes: a patient-centered approach. Position statement of the American Diabetes Association (ADA) and the European Association for the Study of Diabetes (EASD). Diabetes Care. 2012;35:1364-79.

3. Bandura A. Self-efficacy: toward a unifying theory of behavioral change. Psychol Rev. 1977;84:191-215.

4. Tripp-Reimer T, Choi E, Skemp Kelley L, Enslein JC. Cultural barriers to care: inverting the problem. Diabetes Spectrum. 2001;14:13-22.

5. Glasgow RE, Toobert DJ, Gillette CD. Psychosocial barriers to diabetes self-management and quality of life. Diabetes Spectrum. 2001;14:33-41.

6. Coonrod BA. Overcoming physical barriers to diabetes self-care: reframing disability as an opportunity for ingenuity. Diabetes Spectrum. 2001;14:28-32.

7. Nam S, Chesla C, Stotts NA, Kroon L, Janson SL. Barriers to diabetes management: patient and provider factors. Diabetes Res Clin Pract. 2011;93:1-9.

8. Wing RR. Long-term effects of a lifestyle intervention on weight and cardiovascular risk factors in individuals with type 2 diabetes mellitus: 4-year results of the Look AHEAD trial. Arch Intern Med. 2010;170:1566-75.

9. Kavookjian J. Motivational interviewing. Science and practice of pharmacotherapy I and IIPSAP-VII, Book 8. Lenexa: American College of Clinical Pharmacy; 2011. p. 1-18.

10. Miller WR, Rollnick S. Motivational interviewing: preparing people for change. New York: Guilford Press; 2002.

11. Song $\mathrm{D}, \mathrm{Xu} \mathrm{TZ}$, Sun QH. Effect of motivational interviewing on self-management in patients with 
type 2 diabetes mellitus: a meta-analysis. Int J Nurs Sci. 2014;1:291-7.

12. Prochaska J, Norcross J. Systems of psychotherapya transtheoretical analysis. New York: Wadsworth Pub Co; 2003.

13. Elder JP, Ayala GX, Harris S. Theories and intervention approaches to health-behavior change in primary care. Am J Prev Med. 1999;17: 275-84.

14. Johnson SS, Paiva AL, Cummins CO, Johnson JL, Dyment SJ, Wright JA, et al. Transtheoretical model-based multiple behavior intervention for weight management: effectiveness on a population basis. Prev Med. 2008;46:238-46.

15. Ruggiero L. Helping people with diabetes change behavior: from theory to practice. Diabetes Spectrum. 2000;13:125-31.

16. Prochaska JO, Velicer WF. The transtheoretical model of health behavior change. Am J Health Promot. 1997;12:38-48.

17. Jones H, Edwards L, Vallis TM, Ruggiero L, Rossi SR, Rossi JS, et al. Changes in diabetes self-care behaviors make a difference in glycemic control: the diabetes stages of change (DiSC) study. Diabetes Care. 2003;26:732-7.

18. Graffigna G, Barello S, Libreri C, Bosio CA. How to engage type-2 diabetic patients in their own health management: implications for clinical practice. BMC Public Health. 2014;14:648.

19. Blake CE, Hebert JR, Lee DC, Adams SA, Steck SE, Sui $X$, et al. Adults with greater weight satisfaction report more positive health behaviors and have better health status regardless of BMI. J Obes. 2013;2013:291371.

20. Traina S, Guthrie R, Slee A. The impact of weight loss on weight-related quality of life and health satisfaction: results from a trial comparing canagliflozin with sitagliptin in triple therapy among people with type 2 diabetes. Postgrad Med. 2014;126:7-15.

21. Gabler NB, Duan N, Liao D, Elmore JG, Ganiats TG, Kravitz RL. Dealing with heterogeneity of treatment effects: is the literature up to the challenge? Trials. 2009; $10: 43$.

22. Cefalu WT, Leiter LA, Yoon K-H, Arias P, Niskanen L, Xie J, et al. Efficacy and safety of canagliflozin versus glimepiride in patients with type 2 diabetes inadequately controlled with metformin (CANTATA-SU): 52 week results from a randomised, double-blind, phase 3 non-inferiority trial. Lancet. 2013;382:941-50.
23. Leiter LA, Yoon K-H, Arias P, Langslet G, Xie J, Balis $D$, et al. Canagliflozin provides durable glycemic improvements and body weight reduction over 104 weeks versus glimepiride in patients with type 2 diabetes on metformin: a randomized, double-blind, Phase 3 study. Diabetes Care. 2015;38:355-64.

24. Kolotkin RL, Crosby RD, Kosloski KD, Williams GR. Development of a brief measure to assess quality of life in obesity. Obes Res. 2001;9:102-11.

25. Talbot F, Nouwen A, Gingras J, Gosselin M, Audet J. The assessment of diabetes-related cognitive and social factors: the Multidimensional Diabetes Questionnaire. J Behav Med. 1997;20:291-312.

26. Lane S, Levy AR, Mukherjee J, Sambrook J, Tildesley $\mathrm{H}$. The impact on utilities of differences in body weight among Canadian patients with type 2 diabetes. Curr Med Res Opin. 2014;30:1267-73.

27. Bolin JH, Edwards JM, Finch WH, Cassady JC. Applications of cluster analysis to the creating of perfectionism profiles: a comparison of two clustering approaches. Front Psychol. 2014;5:1-9.

28. Wilding JP, Charpentier G, Hollander $P$, Gonzalez-Galvez G, Mathieu C, Vercruysse F, et al. Efficacy and safety of canagliflozin in patients with type 2 diabetes mellitus inadequately controlled with metformin and sulphonylurea: a randomised trial. Int J Clin Pract. 2013;67:1267-82.

29. Lavalle-González FJ, Januszewicz A, Davidson J, Tong C, Qiu R, Canovatchel W, et al. Efficacy and safety of canagliflozin compared with placebo and sitagliptin in patients with type 2 diabetes on background metformin monotherapy: a randomised trial. Diabetologia. 2013;56:2582-92.

30. Schernthaner G, Gross JL, Rosenstock J, Guarisco M, $\mathrm{Fu} \mathrm{M}$, Yee J, et al. Canagliflozin compared with sitagliptin for patients with type 2 diabetes who do not have adequate glycemic control with metformin plus sulfonylurea: a 52-week, randomized trial. Diabetes Care. 2013;36:2508-15.

31. Ajzen I. The theory of planned behavior. Organizational and Human Decision Processes. 1991;50:179-211.

32. Ware JE. SF-36 v2 ${ }^{\circledR}$ Health Questionnaire: Administration Guide for Clinical Trial Investigators. 2008.

33. Polonsky WH. Emotional and quality-of-life aspects of diabetes management. Curr Diab Rep. 2002;2:153-9.

34. Waugh N, Cummins E, Royle P, Clar C, Marien M, Richter B, et al. Newer agents for blood glucose 
control in type 2 diabetes: systematic review and economic evaluation. Health Technol Assess. 2010;14:1-248.

35. Feldstein AC, Nichols GA, Smith DH, Stevens VJ, Bachman K, Rosales AG, et al. Weight change in diabetes and glycemic and blood pressure control. Diabetes Care. 2008;31:1960-5.

36. Feldstein AC, Nichols GA, Smith DH, Rosales AG, Perrin N. Weight change and glycemic control after diagnosis of type 2 diabetes. J Gen Intern Med. 2008;23:1339-45.
37. Walraven I, Mast MR, Hoekstra T, Jansen AP, van der Heijden AA, Rauh SP, et al. Distinct HbA1c trajectories in a type 2 diabetes cohort. Acta Diabetol. 2015;52:267-75. 